\title{
Observations on distal intravascular pressure changes and cerebral blood flow after common carotid artery ligation in man
}

\author{
A. E. HOLMES, I. M. JAMES ${ }^{1}$, AND C. C. WISE 2 \\ From the Departments of Neurological Surgery and Neurology and Anaesthetics, \\ Addenbrooke's Hospital, Cambridge
}

SUMMARY Studies of cerebral blood flow and distal intravascular pressure changes were studied in 11 patients at the time of common carotid artery ligation for the treatment of intracranial aneurysm. No neurological deterioration was observed while these patients were under observation. Cerebral blood flow in the ipsilateral hemisphere recovered rapidly while reduction in pressure, and in particular diminution of pulse pressure, persisted affording protection to the aneurysm.

Ligation of the common carotid artery is a widely used method for the treatment of intracranial aneurysms particularly of the internal carotid artery. It is important to assess the risk of neurological sequelae that follow the occlusion. Precautions are taken to confirm cross-filling at angiography, and trial occlusion by Matas's test and at operation have not reduced the general incidence of neurological complications below $10 \%$. Jennett, Harper, and Gillespie (1966) demonstrated the usefulness of measuring cerebral blood flow in the ipsilateral hemisphere and suggested that the likelihood of developing neurological sequelae is increased if cerebral blood flow is reduced by more than $25 \%$ of the resting level. Pressure measurements in the distal vessels by Bakay and Sweet (1953) and Odom, Woodhall, Tindall, and Jackson (1962) demonstrated the benefits of the ligation with regard to the aneurysm itself. Monitoring the intravascular pressure is another safeguard during the initial period after ligation.

We are reporting here a series of 11 patients in which both these factors were studied simultaneously with a view to establishing their relationship to the neurological status.

'Present address: Department of Therapeutics, Royal Free Hospital. 'Present address: Department of Anaesthetics, Cardiff Royal Infirmary.
PATIENTS

In this investigation 11 patients between the ages o 20 and 63 were studied at the time of elective surger for treatment of aneurysms on the internal carotic artery. One was on the intracavernous part, six d the origin of the posterior communicating artery, and four at the bifurcation of the internal carotid artery. There were seven females. All had suffered from proven subarachnoid haemorrhages and had been investigated by bilateral carotid arteriography with, in some cases, vertebral arteriography. Good cross-filling had been demonstrated at angiography in all cases. In patients who had multiple aneurysms, the neurological signs and focal features in the angiograms suggested that it was the internal carotid artery aneurysm that had bled. The earliest attempt at ligation was on the fifth day, the latest on the 31st day after the last subarachnoid haemorrhage. No neurological disturbance was caused in any patient during the 30 minute trial period, when a temporary Crile's clamp was on the common carotid artery, and it was possible in all cases to convert temporary occlusion into definitive ligation. Only one patient showed any subsequent neurological sequel and that was a mild contralateral hemiparesis, which began 48 hours afterwards, and had completely cleared after a few days. All the patients were well with no neurological deficit attributable to the ligation when they were seen at routine follow-up appointments at 
periods from six months to 33 months afterwards. Postoperative angiography was not performed as routine.

METHOD

The patients were premedicated with droperidol and phenoperidine, according to weight, and were fully conscious to assist with neurological assessment during the 30 minute period of temporary occlusion. Local analgesia, without adrenaline, was used for infiltration of the skin of the neck and a transverse skin crease incision at the level of the hyoid bone was used to allow adequate exposure of the terminal part of the common carotid artery and the commencement of the internal and external carotid vessels. The site on the common carotid artery for application of the Crile's clamp was prepared. A flexible 18 gauge Teflon arterial cannula 4 in. $(8 \cdot 8 \mathrm{~cm})$ long was introduced into the internal carotid artery and a similar arterial cannula was inserted percutaneously into the left brachial artery. Arterial pressures were monitored continuously by strain gauge transducers and recorded on twin channel pen recorders. The cerebral blood flow measurements were made using the ${ }^{133}$ Xenon dilution technique (Ingvar and Lassen, 1961). Approximately $25 \mu \mathrm{c}^{133}$ Xenon in $20 \mathrm{ml}$. normal saline was injected slowly to achieve a constant level of irradiation over 100 to 120 seconds. A wide angle thallium activated sodium iodide crystal detector was used. The duration of the cerebral blood flow measurement was about 15 minutesflow being calculated from the slope of the decay curve which was separated into fast and slow components representing blood flow in the grey matter and white matter respectively.

Measurements of systemic (left brachial artery) and internal carotid artery blood pressures were made in resting control conditions. Recordings were also made as the Crile's clamp was being closed on the common carotid artery and again at $\mathbf{1 5}$ and $\mathbf{3 0}$ minutes after closure.

Cerebral blood flow measurements were made under the control conditions, again within a few minutes of closure of the temporary clamp and finally after an interval of 30 minutes temporary occlusion. Arterial carbon dioxide tensions were measured at intervals with a Micro-Astrup apparatus.

The effect on the systemic and internal carotid artery blood pressures of short periods of occlusion, from 10 to 20 seconds, of the external carotid artery was studied both before and after occlusion of the common carotid artery.

\section{RESULTS}

The systolic pressure in the internal carotid artery fell immediately to a mean level of $49 \%$ of the control, but had recovered at the end of 30 minutes to $62 \%$ of the control level, most of this recovery occurring early during this period. The systemic pressure showed little change. The diastolic pressure in the internal carotid artery also fell, but to a lesser extent, being $62 \%$ immediately after clamping and recovering to $75 \% 30$ minutes later. The pulse pressure showed a much greater decrease, and immediately after clamping had fallen to $18 \%$ of its previous level and at the end of half an hour had recovered only to $33 \%$ of the control figure (Fig. 1).
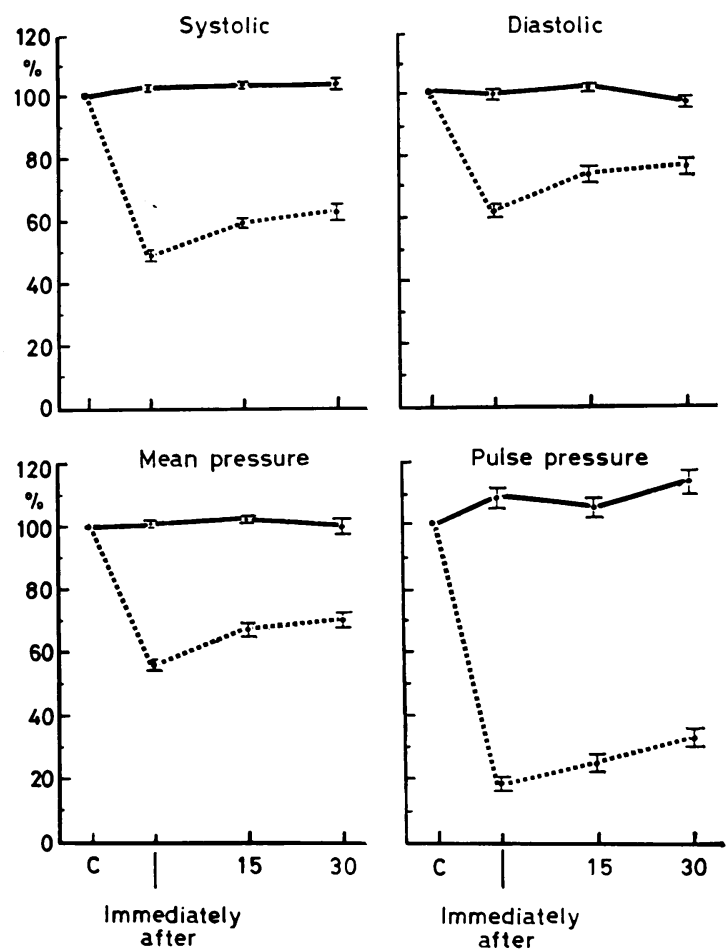

Minutes after clamping

FIG. 1. Effects of common carotid occlusion on blood pressure in internal carotid artery. Blood pressure expressed as percentage of control. Solid lines denote systemic blood pressure. Dotted lines denote internal carotid blood pressure. $C=$ control. $\mathrm{I}=$ means and standard error of the means.

The effect on cerebral blood flow showed a more rapid recovery than that for blood pressure, and the cerebral blood flow grey(CBFG) fell from $65 \mathrm{ml} . / 100 \mathrm{~g}$ $/ \mathrm{min}$ to $51 \mathrm{ml} . / 100 \mathrm{~g} / \mathrm{min}$ shortly after clamping and by the end of the 30 minute period had almost completely recovered to the resting level. The cerebral blood flow white (CBFW) showed an early fall from $22 \mathrm{ml} . / 100 \mathrm{~g} / \mathrm{min}$ to $16 \mathrm{ml} . / 100 \mathrm{~g} / \mathrm{min}$ immediately after clamping, and this too had completely recovered at the end of 30 minutes (Fig. 2).

The results of temporary occlusion of the external carotid artery were assessed by change in pressure 

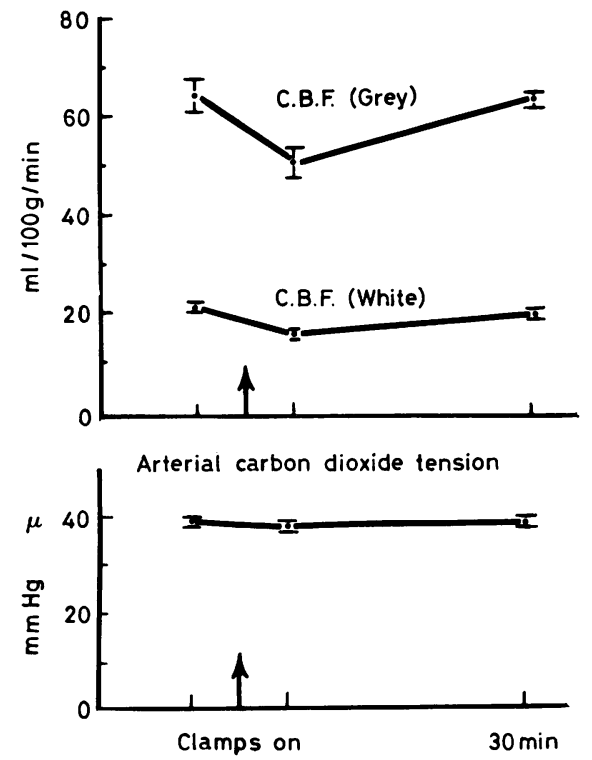

FIG. 2. Effect of common carotid artery occlusion on cerebral blood flow in the ipsilateral hemisphere. (Excluding patient with extracranial flow pattern.)

in the internal carotid artery and demonstrated that in only one case was there any increase in pressure when the clamp was applied. This was taken to represent a retrograde flow down the internal carotid artery and into the external carotid artery. This observation was supported by disturbances in the cerebral blood flow measurement which suggested that a clearance curve from the extracerebral tissue was being obtained.

\section{DISCUSSION}

Ligation of the common carotid artery causes marked changes in the intravascular pressure and flow in the internal carotid artery beyond. Black and German (1953) have discussed several factors that influence the circulatory haemodynamics of an aneurysm-namely, hydrostatic pressure, pulsatile flow, turbulence, and jet action. The present study demonstrated that the disturbance of pressure and flow was maximal at the time of occlusion. The most marked change was seen in the reduction of the pulse pressure to only a fifth of its previous level. This reduction of pulsation of an aneurysm is an important factor in reducing aneurysmal enlargement and the likelihood of rupture. The reduction of intra-aneurysmal hydrostatic pressure is also important; the discrepancy between common carotid artery and intra-aneurysmal pressure for internal $\underset{\mathbb{Q}}{Z}$ carotid artery aneurysms is likely to be small. Indeed, $\stackrel{ }{\bar{D}}$ Bakay and Sweet (1953) have found intravascular of pressure in major vessels to be comparable with $\frac{C}{0}$ pressure in the more peripheral branches and 0 recently Wright (1968) has demonstrated that intraaneurysmal pressure is also similar to that in the internal carotid artery.

Our interest was particularly concerned with the comparison between the complete recovery of the $\overrightarrow{\vec{F}}$ cerebral blood flow and the partial recovery of the internal carotid artery pressure. This means that the disturbance of perfusion recovers rapidly, but that the beneficial effects of lowered hydrostatic and $\vec{\sigma}$ lowered pulse pressure after common carotid artery $\varnothing$ ligation afford some protection to an aneurysm and may well induce changes that cause obliteration of $\vec{\circ}$ the lumen and more permanent healing. Our studies covered only the acute period, but Youmans, Kindt, and Mitchell (1967), while investigating the duration of reversed flow in the external carotid artery, carried out studies that followed the recovery of pressure over periods up to several days. They found a $\$$ moderate acute reduction of mean blood pressure to․ $72 \%$ of the preocclusion pressure and a marke $\infty$ reduction of pulse pressure to $20 \%$ of the pres occlusion pulse pressure. Both of these pressure $\vec{P}$ showed a return towards normal levels in theio delayed studies over several days, but pulse pressure remained reduced to $41 \%$ of preocclusion levels. The acute fall of pulse pressure in our patients was of a comparable degree. Odom et al. (1962), in a series of 31 patients who had late angiography to examine the long-term effects of common carotid artery ligation on the angiographic size of aneurysms, extended their studies from nine days to 34 months after ligation. They showed that there was a gradual return of distal intravascular pressure towards normal levels and even with widely differing 'immediate' reductions the 'late' pressures above the site of ligation were similar-between $63 \%$ and $75 \%$ of the original preocclusion pressures. Cristiansson (1962), studying the late postoperative effects by a measurement of retinal artery pressure using ophthalmodynamometry in 13 patients with carotid artery ligation, found that eight of these had pressure drops of more than $20 \%$ when assessed at one to 13 years after ligation.

In one of our patients the common carotid clamp was released a few minutes after clamping and, although the internal carotid artery pressure immediately achieved the control levels, reapplication of the clamp after 20 seconds showed that the curve of recovery had continued during the interval. This indicates that the mechanisms involved in the establishment of collateral circulation continue their re- 
adjustment during the time of temporary release of the clamp.

The effect of clamping the external carotid artery on the pressure in the internal carotid artery after occlusion of the common carotid artery gives some indication of the direction of blood flow. Sweet and Bennett (1948) established that the flow was not necessarily always down the external carotid artery and forwards in the internal carotid artery as had previously been suggested by Dorrance (1934). Hardesty, Roberts, Toole, and Royster (1961) and Tindall, Odom, Dillon, Cupp, Mahaley, and Greenfield (1963), using electromagnetic flowmeters, have demonstrated the flow changes that were predicted by the pressure gradients observed. The direction of flow can give some indication of the relative effectiveness of the internal or external collateral circulation. This manoeuvre was carried out in all our patients during the control period and immediately after temporary occlusion and at the end of the 30 minute period of trial clamping. There was retrograde flow in the internal carotid artery as judged by the pressure changes and confirmed by flow measurements in only one patient. This had not reversed by the end of the 30 minute period of trial clamping. Hardesty et al. (1961) had found the retrograde flow in the internal carotid artery in seven of 15 patients studied. These were investigated at the time of treatment for malignancy of the head and neck and did not have intracranial aneurysms. Further studies of Youmans et al. (1967) have shown that this effect is relatively short lived and all six of the patients they studied, who had reversal of the flow immediately after clamping, had reverted to forward flow in the internal carotid artery within 14 hours; three of them did so within 30 minutes, two within three hours, and the other by 14 hours.

Most of the studies performed on blood flow changes at the time of carotid ligation have been done on the vessels of the neck. This does not necessarily reflect the state of affairs in the ipsilateral hemisphere. Our findings of a moderate, short-lived reduction of cerebral blood flow in the ipsilateral hemisphere demonstrate that the adjustments of perfusion occur more rapidly than the adjustments of pressure. At the end of the trial period of clamping of 30 minutes the cerebral blood flow had returned to normal, while the beneficial effects of greatly reduced pulse pressure and moderately reduced hydrostatic pressure remained.

During this study we did not encounter any patient who developed neurological deterioration in the operative period and consequently we were not able to find a level at which we could say that it would be unwise to proceed with permanent ligation. None of them had a fall of cerebral blood flow as great as $25 \%$, which Jennett et al. (1966) have suggested leads to development of hemiparesis, and we therefore see no cause to modify this figure. The timing of the ligation is an important factor in avoiding the risk of causing neurological complications and it is worth noting that none of these patients was operated on less than five days from the time of their subarachnoid haemorrhage.

We are grateful to Mr. Walpole Lewin and Mr. John Gleave for their helpful criticism during this study.

\section{REFERENCES}

Bakay, L., and Sweet, W. H. (1953). Intra-arterial pressures in the neck and brain. Late changes after carotid closure, acute measurements after vertebral closure. $J$. Neurosurg., 10, 353-359.

Black, S. P. W., and German, W. J. (1953). The treatment of internal carotid artery aneurysms by proximal arterial ligation. A follow-up study. J. Neurosurg., 10, 590-601.

Cristiansson, J. (1962). On the late effects of carotid ligation upon the human eye. Acta ophthal. (Kbh.), 40, 271-280.

Dorrance, G. M. (1934). Ligation of the great vessels of the neck. Ann. Surg., 99, 721-742.

Hardesty, W. H., Roberts, B., Toole, J. F., and Royster, H. P. (1961). Studies on carotid artery flow. Surgery, 49, 251-256.

Ingvar, D. H., and Lassen, N. A. (1961). Quantitative determination of regional cerebral blood-flow in man. Lancet, 2, 806-807.

Jennett, W. B., Harper, A. M., and Gillespie, F. C. (1966). Measurement of regional cerebral blood-flow during carotid ligation. Lancet, 2, 1162-1163.

Odom, G. L., Woodhall, B., Tindall, G. T., and Jackson, J. R. (1962). Changes in distal intravascular pressure and size of intracranial aneurysm following common carotid ligation. J. Neurosurg., 19, 41-50.

Sweet, W. H., and Bennett, H. S. (1948). Changes in internal carotid pressure during carotid and jugular occlusion and their clinical significance. $J$. Neurosurg., 5, 178-195.

Tindall, G. T., Odom, G. L., Dillon, M. L., Cupp, H. B., Jr., Mahaley, M. S., Jr., and Greenfield, J. C., Jr. (1963). Direction of blood flow in the internal and external carotid arteries following occlusion of the ipsilateral common carotid artery. Observation in 19 patients. J. Neurosurg., 20, 985-994.

Wright, R. L. (1968). Intraaneurysmal pressure reduction with carotid occlusion. Observations in three cases of middle cerebral aneurysms. J. Neurosurg., 29, 139-142.

Youmans, J. R., Kindt, G. W., and Mitchell, O. C. (1967). Extended studies of direction of flow and pressure in the internal carotid artery following common carotid artery ligation. J. Neurosurg., 27, 250-254. 